\title{
INTERACTING SOLITARY WAVES IN A DAMPED DRIVEN LENNARD-JONES CHAIN
}

\author{
Theo P. VALKERING and Joost H.J. VAN OPHEUSDEN \\ Center for Theoretical Physics, Twente University of Technology, P.O. Box 217, 7500 AE Enschede, The Netherlands
}

\begin{abstract}
It is shown analytically that pulse solitary waves in a chain with Lennard-Jones type nearest neighbor interaction are strongly localized and marginally stable in the high energy limit.

In a damped and periodically driven chain we obtain numerically families of states whose behavior is similar to that of equally many oscillators. We observe a period doubling sequence in a one-solitary wave family and bifurcation to (quasi-) periodic motion in a family of two solitary waves. We conclude that the damped and driven chain admits asymptotically stable states living on a low-dimensional manifold in phase space. These results depend sensitively on the shape of the driving term.
\end{abstract}

\section{Introduction}

Pulse solitary waves in several different chains of nonlinearly coupled particles have the common properties i) that they are strongly localized at high energy, ii) that they are effectively stable for small perturbations and iii) that they remain intact after collision with each other [1-3]. Because of these properties one might guess that in a damped and properly driven chain asymtotic states exist which consist of a single, or a few interacting solitary waves. Similar states have been observed in perturbed sine-Gordon systems [4-8], where the interaction can lead to low dimensional temporal chaos.

The aim of this paper is twofold. First we establish analytically the localization and stability of waves in a free and undamped chain with nearest neighbor interaction on the Lennard-Jones type, in the limit of high energy. To obtain this result it is pointed out that a pulse solitary wave in a chain with periodic boundary conditions is a solution of a constrained variational problem: it represents a maximum of the time averaged potential energy for constant kinetic energy and period, with respect to variations in the class of traveling waves. Analytical investigation of this problem demonstrates that the wave in the limit of high energy is a sharp pulse-like wave, with width equal to one particle distance and infinite phase velocity. Furthermore this wave is marginally stable. Its Floquet multipliers are calculated. They are on the unit circle and have generalized eigenspace of dimension two.

Secondly we investigate numerically a damped and periodically driven chain. We find separate asymptotic states consisting of one, two or three solitary waves. If the driving term is varied properly, these states show bifurcations similar to those in systems of equally many driven oscillators.

In particular we consider a damped chain with one end particle fixed and the other undergoing a prescribed periodic motion. We describe one state (cf. fig. 2) in which one solitary wave bounces to and fro between the two end particles with the period of the driving term. This state undergoes a sequence of period doublings, similar to those of the bouncing ball (Fermi) problem [9].

We also show a state (cf. fig. 3) consisting of two solitary waves with equal period. This state, modeled by two interacting identical oscillators, shows a transition from a periodic to a (quasi-) periodic state (on a 2-torus) through a Hopf bifurcation [10]. In addition we found states with three solitary waves, showing similar bifurcations.

On the basis of these results we conjecture that the present chain admits asymptotic states living on a low-dimensional manifold in phase space, 
characterized by a just few solitary waves. Furthermore our results indicate that temporal chaos can occur on this manifold, however only for a carefully chosen driving term. A sinusoidal driving term generally will not work (cf. [18, 19] for similar work on the Toda chain).

\section{Stability and localization of solitary waves in a circular chain}

Here we point out that solitary waves in the infinite energy limit are strongly localized. Details are given in [11, 12]. The Floquet multipliers in this limit are calculated analytically and appear to be on the unit circle.

Consider a circular chain of $N$ particles with nearest neighbor interaction potential $V=V\left(r_{n+1}\right.$ $\left.-r_{n}\right)$. This potential satisfies: $V(0)=V^{\prime}(0)=0$, $V^{\prime \prime}(0)>0$ and $r_{n}$ denotes the deviation of the $n$th particle from its equilibrium position. Furthermore we assume that zero is the only extremum and that $V$ has a vertical asymptote at -1 , i.e. $V(x) \rightarrow+\infty$ if $x \rightarrow-1$.

We consider waves of the form $r_{n}(t)=r(\omega t-$ $n k$ ), where $r$ is a $2 \pi$-periodic function of its argument and $k$ denotes $2 \pi / N$. Then $\omega$ denotes the angular frequency. If the function $s(\theta)$ and the linear operator $A$ are defined as

$s(\theta)=\frac{\mathrm{d} r}{\mathrm{~d} \theta}(\theta), \quad A s(\theta)=-\int_{-k / 2}^{+k / 2} s\left(\theta^{\prime}\right) \mathrm{d} \theta^{\prime}$,

one readily verifies that the velocities and displacements are

$\dot{r}_{n}(t)=\omega s(\omega t-n k)$,

$r_{n+1}(t)-r_{n}(t)=(A s)\left(\omega t-n k-\frac{1}{2} k\right)$.

The time average per particle of the potential and of the kinetic energy for such a wave depend on $s$ and $\omega$ and read respectively

$$
\begin{aligned}
& \mathscr{V}(s)=\frac{1}{2 \pi} \int_{-\pi}^{\pi} V(A s(\theta)) \mathrm{d} \theta, \\
& \mathscr{T}(\omega, s)=\omega^{2} \mathscr{N},
\end{aligned}
$$

where

$\mathscr{N}=\frac{1}{2} \frac{1}{2 \pi} \int_{-\pi}^{\pi} s^{2}\left(\theta^{\prime}\right) \mathrm{d} \theta$.

Substitution of the traveling wave solution $r(\omega t-$ $n k$ ) in the Euler-Lagrange equations of motion yields a variational problem for $s$

$\omega^{2} \operatorname{grad} \mathscr{N}(s)=\operatorname{grad} \mathscr{V}(s)$.

Summarizing: a traveling wave solution corresponds to a stationary point of the functional $\mathscr{V}$ for constant $\mathscr{N}$, where $\omega^{2}$ is the Lagrange multiplier. With the expression (2.3) for $\mathscr{T}$ one easily sees that (2.4) is equivalent to the problem: find stationary points of $\mathscr{V}$ for constant $\mathscr{T}$ and $\omega$.

Using the variational problem (2.4) and looking for maxima of $\mathscr{V}$, one derives that a solution $\left\{\omega^{2}(\bar{V}), s(\bar{V})\right\}$ exists [11] for each positive value $\bar{V}$ of this maximum. For low values of $\bar{V}$ this solution is the linear traveling wave with longest possible wavelength: $s \sim \bar{V}^{1 / 2} \cos (\theta)$ [12]. For high values we have, due to the existence of the vertical asymptote at -1 in the interaction potential

Theorem 1. If the integral $\int_{0}^{-1} V(x) \mathrm{d} x$ of the interaction potential diverges, then the solution $\left\{\omega^{2}(\bar{V}), s(\bar{V} ; \theta)\right\}$ of $(2.4)$ satisfies

$$
\lim _{\bar{V} \rightarrow \infty} \omega^{-2}(\bar{V})=0, \quad \lim _{V \rightarrow \infty} s(\bar{V} ; \theta)=s_{0}(\theta),
$$

where $s_{0}$ is the step function as shown in fig. 1 .

Remark 1. The time dependence of $r_{n}(t)$ and $r_{n+1}(t)-r_{n}(t)$ follows from (2.2) and fig. 1. Clearly it is a motion of hard point masses, where the solitary wave is carried by precisely one particle, which transfers its momentum to the next one etc. Observe that $\left(A s_{0}\right)(\theta)>-1$, except at $\theta=0$, corresponding to $V=\infty$.

Remark 2. Numerical solution of the variational problem (2.4) (for a Lennard-Jones 6-12 poten- 


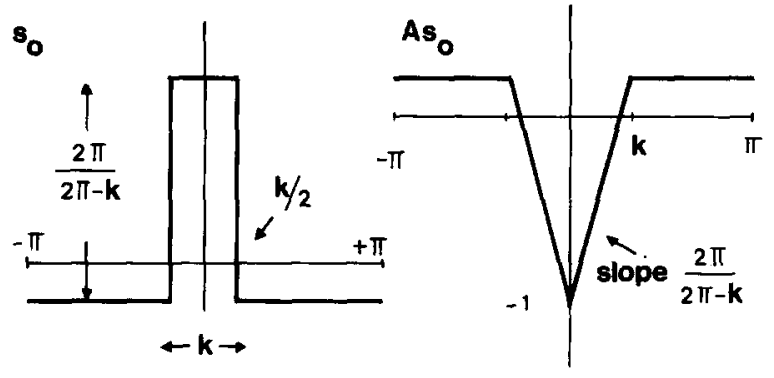

Fig. 1. The functions $s_{0}(\theta)$ and $A s_{0}(\theta)$ which describe the solitary wave at high energy (cf. (2.2)).

tial), is found easily [13]. It demonstrates that the wave evolves continuously from the cos-shape to the $s_{0}$-shape as a function of $\bar{V}$. At the same time one obtains the phase velocity, which appears to be a monotonically increasing function of the energy.

To determine the stability of a solitary wave solution at $\bar{V}=\infty$ we consider approximate solutions of the Euler-Lagrange equations for the chain of the form

$r_{n}(t)=r(\bar{V} ; \omega t-n k)+\xi_{n}(t)$,

$\xi_{n}(t)=\mathrm{e}^{\mathrm{i} \alpha \omega(\bar{V}) t} \rho_{n}(t)$,

where $\rho_{n}(t)$ is a $2 \pi / \omega$-periodic function and $\xi_{n}(t)$ satisfies the linearized equations [10]

$\ddot{\xi}_{n}=-m_{n}\left(\xi_{n}-\xi_{n-1}\right)+m_{n+1}\left(\xi_{n+1}-\xi_{n}\right)$,

where

$m_{n}(t)=V^{\prime \prime}\left(A s\left(\bar{V} ; \omega t-\left(n-\frac{1}{2}\right) k\right)\right)$.

One readily verifies that (2.6) has two solutions for all $\bar{V}$

$\alpha=0, \quad \rho_{n}=\rho_{n+1}=$ constant,

$\alpha=0, \quad \rho_{n}(t)=s(\bar{V} ; \omega t-n k)$.

For the other solutions we have

Theorem 2. When $\bar{V} \rightarrow \infty$ the variational equations (2.6) have apart from the two zero solutions
(2.7) precisely $N-2$ different solutions $\left\{\alpha_{m} \mid m=\right.$ $1, \ldots, N-2\}$ which satisfy

$$
\begin{aligned}
\lim _{V \rightarrow \infty} \alpha_{m}=m(1+1 /(N-1)) & \\
& m=1, \ldots, N-2 .
\end{aligned}
$$

The exponents and the corresponding functions $\rho_{n}(t)$ are calculated in the appendix. The fact that the exponent $\alpha$ is real follows from the fact that the wave in the high energy limit is effectively a hard particle motion.

Remark 3. Solution (2.7b) corresponds to a phase shift of the wave. A second solution to this zero eigenvalue is

$\rho_{n}(t)=r_{\bar{v}}(\omega t-n k)+s(\omega t-n k) \omega_{\bar{\nu}} t$,

where the subscript $\bar{V}$ denotes differentiation with respect to $\bar{V}$. This solution corresponds to a shift in $\bar{V}$. Similarly the eigenvalue zero in $(2.7 \mathrm{a})$ and the $N-2$ eigenvalues in (2.8) have generalized eigenspaces of dimension two, i.e. the wave is marginally stable.

Remark 4. Assuming continuity as function of $\bar{V}$, this result shows that $|\operatorname{Im} \alpha|$ can be made small for $\bar{V}$ sufficiently large, i.e. the wave appears to be effectively stable for small perturbations during a large number of periods, if the energy is sufficiently high. This result confirms the numerically observed stability. Though we did not derive it explicitly, one must expect that similar stability and localization results hold for a solitary wave moving to and fro between two fixed walls.

\section{3. n-Solitary wave states in a damped driven chain}

Consider a chain of $N+2$ particles, damping constant $G$ and nearest neighbor interaction potential

$$
\begin{aligned}
& V\left(r_{n+1}-r_{n}\right)=\left(\left(1+r_{n+1}-r_{n}\right)^{-12}\right. \\
& \left.-2\left(1+r_{n+1}-r_{n}\right)^{-6}+1\right) / 12
\end{aligned}
$$


One end particle is held fixed, and the other undergoes a prescribed periodic motion, i.e. the equations of motion

$$
\begin{array}{r}
\ddot{r}_{n}=V^{\prime}\left(r_{n+1}-r_{n}\right)-V^{\prime}\left(r_{n}-r_{n-1}\right)-G \dot{r}_{n}, \\
n=1, \ldots, N,
\end{array}
$$

are integrated with the boundary conditions

$r_{0}(t)=\min \{M, A(1-|\cos (\pi t / P)|)\}$,

$r_{N+1}(t)=0$.

$M$ is a positive parameter which determines the maximal compression of the chain; $A$ determines the curvature of the nonhorizontal part of $r_{0}(t) ; P$ is the period. We consider the behavior of the different states as a function of $A$ for fixed values of $M, P$ and the damping constant $G$. The period $P$ is chosen much smaller than the period of the linear normal mode with largest wavelength. Consequently the observed solitary waves are sharply peaked (cf. section 2).

We focus on three families of states, with $A$ as the parameter, consisting of one or two solitary waves ( $1-\mathrm{s}$ and $2-\mathrm{s}$ families respectively).

Family $i$ ). The family of fig. 2 shows one solitary wave that undergoes the first bifurcations of a period doubling sequence. The values of the fixed parameters are $G=3.0, M=1.2$ and $P=0.1$.

Starting from an initial condition $\dot{r}_{1}=a>0$, $\dot{r}_{n}=0$ if $n \geq 2$, and $r_{n}=0$ where $a$ is properly chosen and $A=1.0$, the system readily stabilizes into a state in which one soliton bounces to and fro in the chain with period equal to the driving period. Changing $A$ slowly from $A=1.0$ to $A=2.0$ period doublings are observed in the total energy of the system and in the spectrum of $r_{1}(t)$.

The bifurcation behavior of this family is very similar to that of the ball, bouncing between a fixed and a moving wall [9]. The velocity of an undamped solitary wave between two fixed walls depends on a parameter as for instance the energy.
Here we observe that the energy shows the period doublings (cf. fig. 2i). We believe that the bifurcation sequence can be completed by modifying the shape of $r_{0}(t)$ at the same period $P$, or doing the same calculations at a smaller value of $P$. This leads us to the conjecture that after the completion of the period doubling sequence, $A>A_{\text {critical }}$, the asymptotic state of our system lives on a two-dimensional surface in phase space.

A nice investigation of a similar phenomenon, namely a soliton in an optical (ring) cavity has been reported in [16]. In [17] a period doubling sequence for a soliton in a perturbed cubic Schrödinger equation was described.

Family iia). The family of fig. 3 shows two interacting solitary waves, each of period one, whose interaction leads to a Hopf bifurcation (fig. 3ii)). The values of the fixed parameters, are $G=0.2$, $M=0.4$ and $P=1.0$. Starting from initial conditions like we did in family i), with $M$ sufficiently large to have no effect, we obtain a $1-\mathrm{s}$ state at $A=0.4$. Increasing $A$ now does not lead to a period doubling sequence but at $A=0.7$ the system passes to a $2-s$ state which retains its character when $A$ is brought back slowly to its original value 0.4 . Finally $M$ is given the value 0.4 as above. Next we observe the bifurcation behavior of this state for $A$ in the range between 0.4 and 2.28. At $A=0.4$ the spectrum shows only sharp peaks at period one and higher harmonics. No changes are observed until at $A=2.2$ a peak appears at period approximately eight. This peak shifts to about nine and back when $A$ increases. This family is interpreted as a combination of two solitary waves on the basis of the $r_{n}$ versus $t$ diagrams. Furthermore its energy equals twice the energy of the corresponding $1-\mathrm{s}$ state (family iib). These results demonstrate that one can find for suitably chosen driving term $r_{0}(t)$ a family of states, similar to a system of two identical driven coupled oscillators.

Family iib). The family of fig. 4 is a $1-\mathrm{s}$ family which coexists with family iia), i.e. $G, M$ and $P$ 

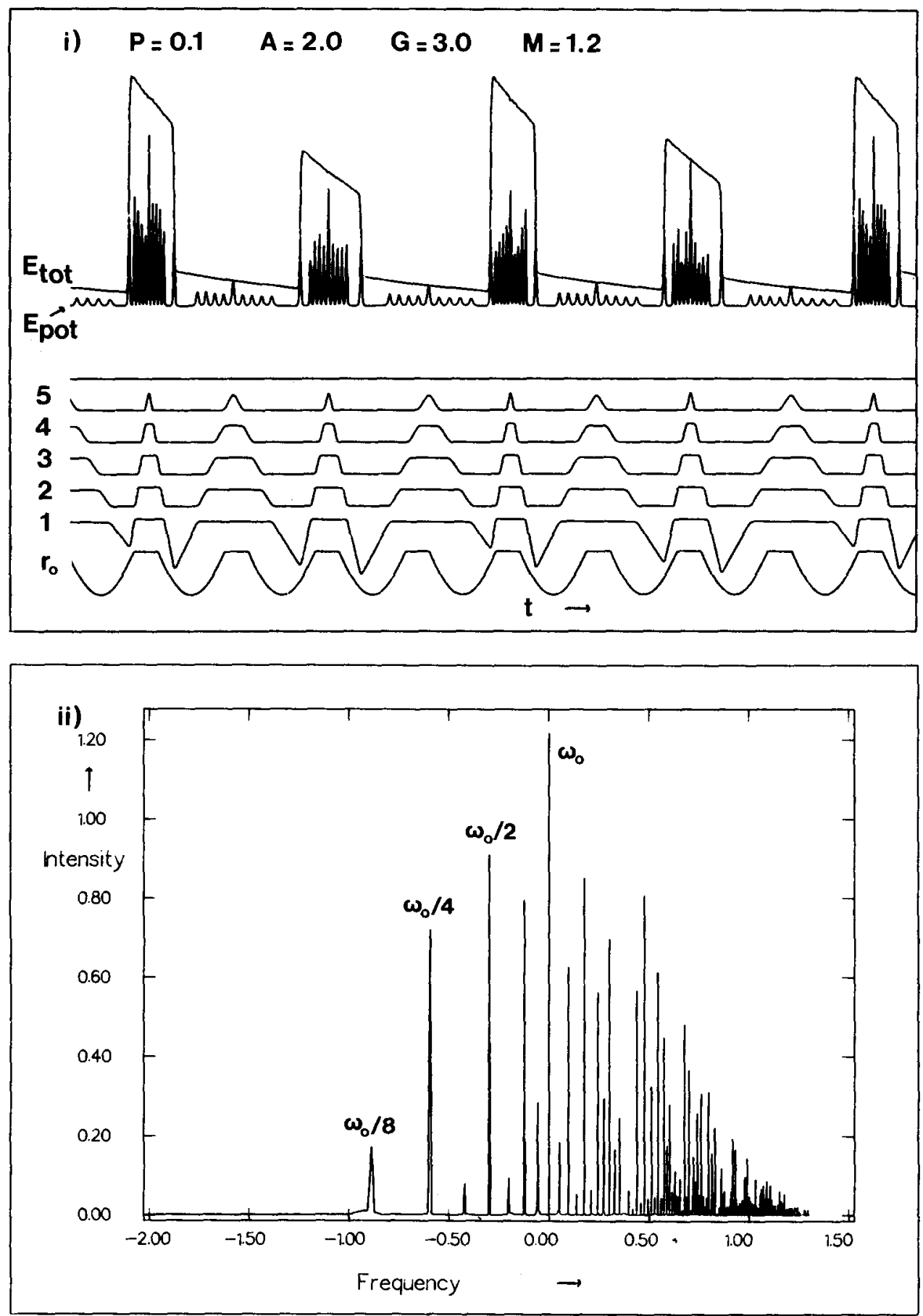

Fig. 2. i) A single solitary wave state of family i) at $A=2.0$ in a chain of five particles, showing period doublings. Plotted as a function of time are the total and potential energy (top) and the positions of the particles including the end particles (bottom). The potential energy shows that the wave propagates through two-particle collisions, as with hard core interaction. Periods two, four and eight are visible in the energy as well as in the wave itself (cf. $\left.r_{1}(t)\right)$. ii) The spectrum of $r_{1}(t)$. Plotted is the intensity log $(1+|a(\omega)|)$ versus $\log \left(\omega / \omega_{0}\right)$ where $a(\omega)$ is the amplitude of the Fourier transform and $\omega_{0}$ is the circular frequency of the driving term. 

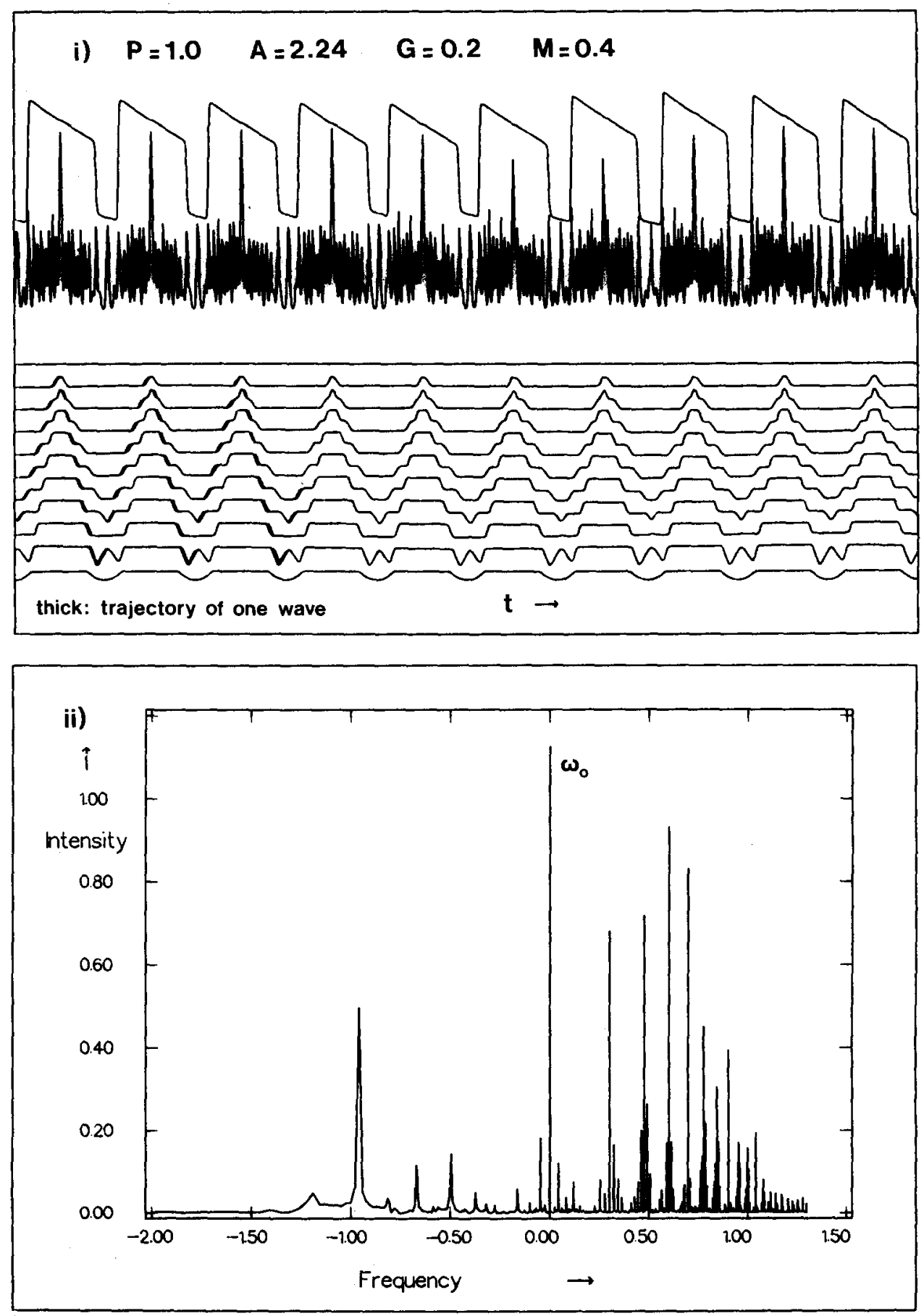

Fig. 3. i) A 2 - s state of family iia) at $A=2.24$ in a chain with nine particles, showing a Hopf bifurcation. A modulation of period about nine appears as a very strong line in the spectrum. This modulation can also be seen in the energy plot, and is also visible in $r_{n}(t)$, just where the two waves interact through a three-particle collision. ii) The peak at $\omega=\omega_{0} / 9$ is due to a Hopf bifurcation of the system of two interacting solitary waves. Its frequency changes when $A$ is varied. The low peaks at higher frequencies can be identified as its higher harmonics. 

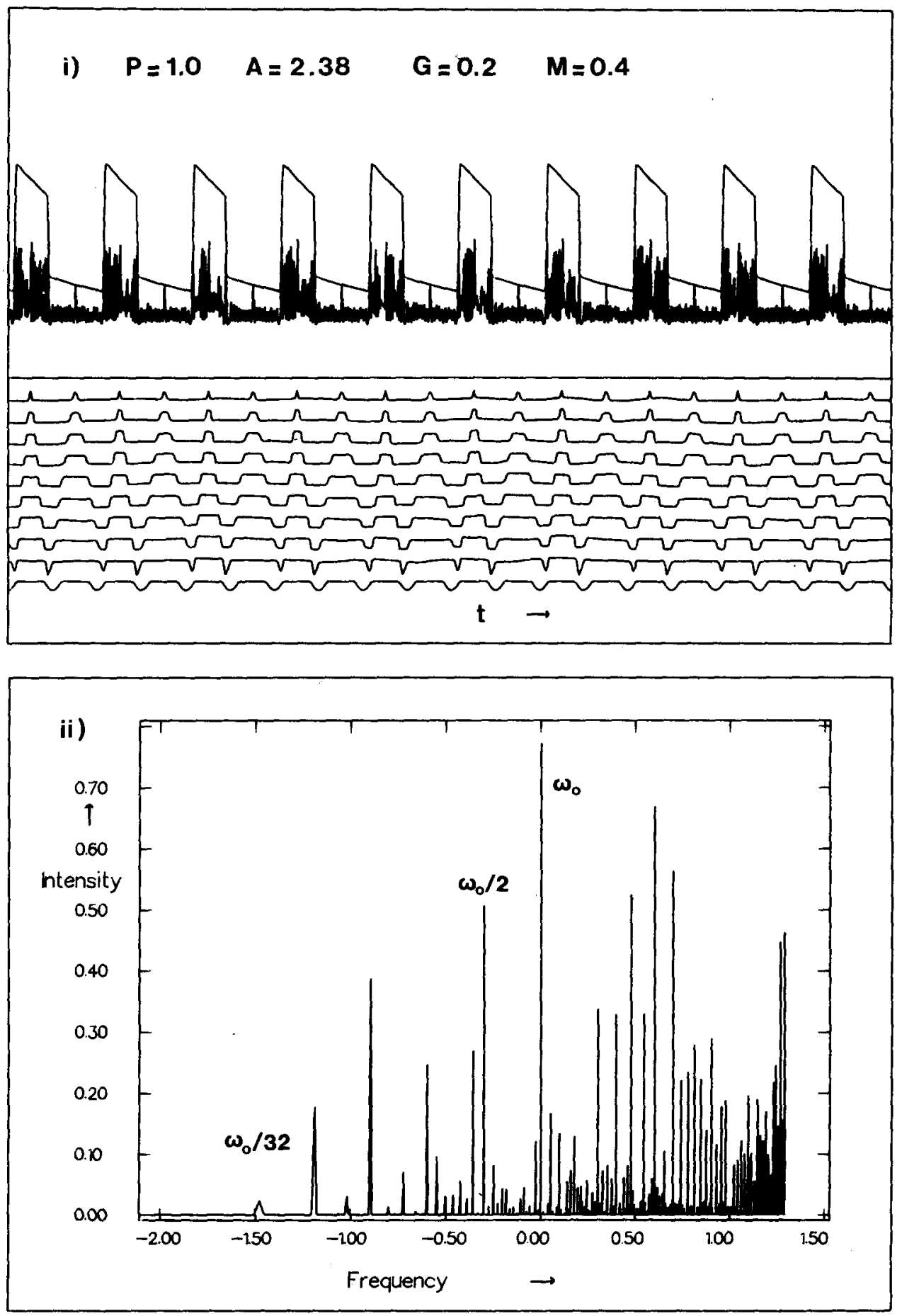

Fig. 4. A single solitary wave of family iib) at $A=2.38$ in the chain of nine particles. This family coexists with the $2-\mathrm{s}$ family of fig. 3. Periods two etc. are apparent in the spectrum and can be seen as modulations on the wave form. In contrast the total energy shows only a period two. We argue that the other subharmonics can be due to the excitation of internal degrees of freedom of the solitary wave. The low peaks at frequencies below $\omega_{0}$ are at integer multiples of the frequency $\omega_{0} / 32$. 


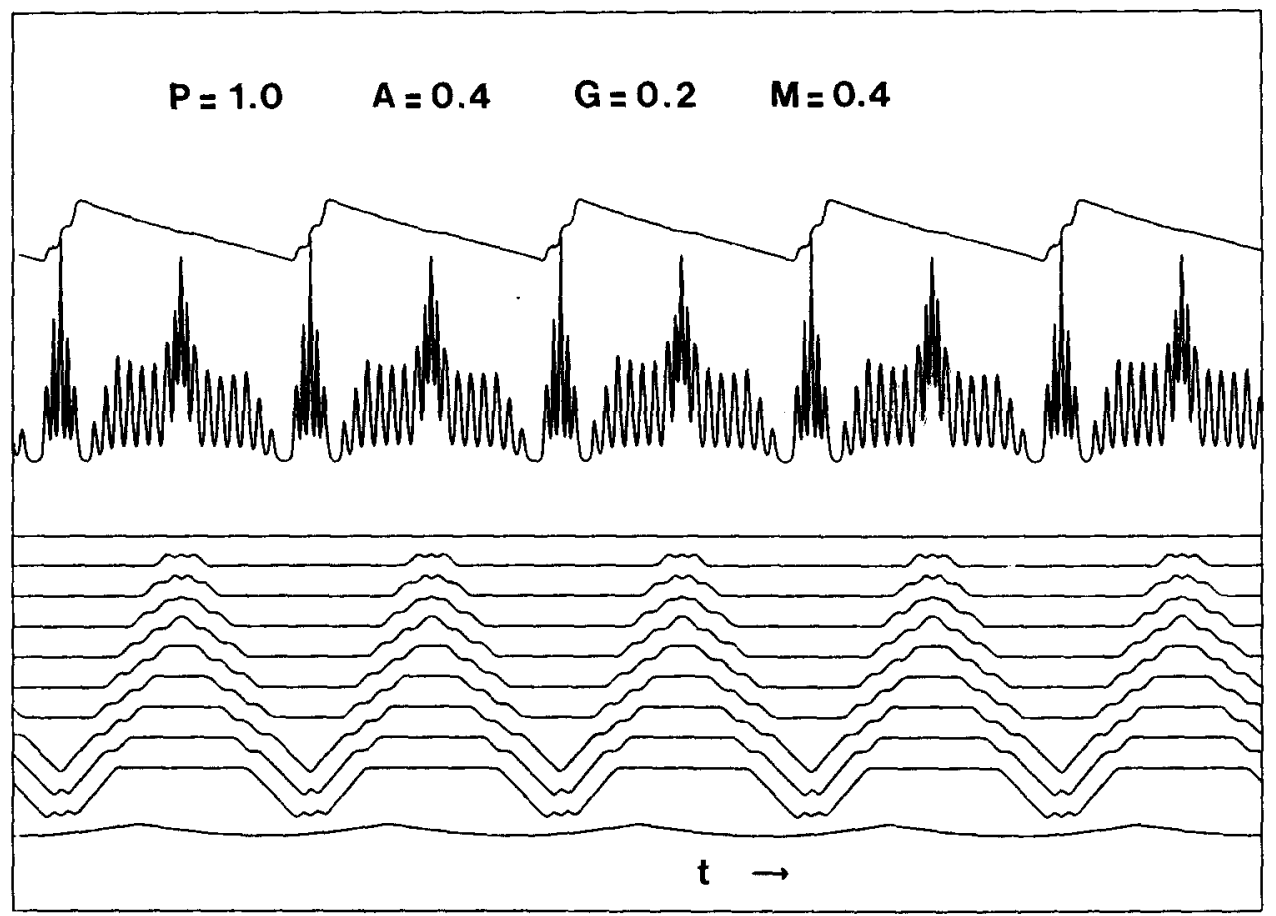

Fig. 5. A $3-s$ state coexisting with the $1-s$ and $2-s$ families of figs. 4 and 3 .

are the same, and the region for $A, 0.4<A<2.4$ overlaps with that of the former.

Changing $A$ from 0.4 to 2.4 we observe a period doubling sequence up to period 32 . The main difference with the $1-s$ family $i$ ) is that the total energy shows only one noticeable period doubling (period two). Higher periods are visible in the motions of the particles only. A possible interpretation is that this state lives on a higher dimensional manifold (as compared with family i)), due to excitation of internal degrees of freedom of the wave.

We also observed $3-\mathrm{s}$ states and bifurcations similar to iia) (cf. fig. 5). Essential for a further study of the $2-\mathrm{s}$ or $3-\mathrm{s}$ families is the understanding of the interaction of two solitary waves. A detailed numerical study of the overtaking collisions in a L-J chain was given in [20]. An interesting analytical investigation of the interaction between kinks was derived in [21]. This method, describing the interaction of the kinks through their radiation modes, could possibly be applied in the present case too. See also $[14,15]$.

We finally remark that our analysis of the $n-s$ states depends for a large part on direct interpretation of the $r_{n}$ versus $t$ diagrams. A more sophisticated way would be to use the canonical transformation to soliton variables for the Toda chain $[22,18]$. Although we are dealing with a different chain common features of high energetic solitons in both chains make it probable that this tool will give useful information in the L-J case as well.

\section{Acknowledgements}

JvO is supported by the 'Stichting voor Fundamenteel Onderzoek der Materie' (F.O.M.). TPV thanks the CNLS at the Los Alamos National Laboratory for enlightening discussions and their hospitality. 


\section{Appendix}

\section{Calculation of the Floquet multipliers}

Substitution of a solution of the form $\rho_{n}(t)=$ $\rho(\omega t-n t)$ in (2.6b) gives when $\omega t-n k$ is replaced by $\theta$

$\left(\mathrm{i} \frac{\mathrm{d}}{\mathrm{d} \theta}-\alpha\right)^{2} \rho(\theta)=-\omega^{-2} B m(\theta) B \rho(\theta)$,

where

$B \rho(\theta)=-\rho\left(\theta+\frac{1}{2} k\right)+\rho\left(\theta-\frac{1}{2} k\right)$,

$m(\theta)=V^{\prime \prime}(A s(\theta))$.

It appears to be useful to perform another substitution, $\rho(\theta)=\mathrm{e}^{-\mathrm{i} \alpha \theta} \sigma(\theta)$, which yields for $\sigma$ the equation

$$
\begin{aligned}
\sigma^{\prime \prime}(\theta)= & \omega^{-2} m\left(\theta+\frac{1}{2} k\right)\left[\mathrm{e}^{\mathrm{i} \alpha k} \sigma(\theta+k)-\sigma(\theta)\right] \\
& -\omega^{-2} m\left(\theta-\frac{1}{2} k\right)\left[\sigma(\theta)-\mathrm{e}^{\mathrm{i} \alpha k} \sigma(\theta-k)\right], \\
\sigma(\theta)= & \mathrm{e}^{\mathrm{i} \alpha \theta} \rho(\theta) .
\end{aligned}
$$

We now look for solutions $\{\alpha, \sigma\}$ such that $\rho(\theta)$ is $2 \pi$-periodic. Essential for the solution are the following limit properties of $\omega^{-2} m(\theta)$,

$$
\begin{aligned}
& \lim _{V \rightarrow \infty} \omega^{-2} m(\theta)=0 \quad \text { if } \quad \theta \neq 0, \\
& \lim _{V \rightarrow \infty} \omega^{-2} \int_{-\varepsilon}^{+\varepsilon} m(\theta) \mathrm{d} \theta \rightarrow \infty, \\
& \lim _{\bar{V} \rightarrow \infty} \omega^{-2} m(\theta) H(\theta)=-\frac{1}{2} \delta^{\prime}(\theta), \quad-\varepsilon<\theta<\varepsilon,
\end{aligned}
$$

where $\delta$ is the Dirac deltafunction, and $H(\theta)$ is the Heaviside function

$H(\theta)=\frac{1}{2}$ if $\theta>0, \quad H(\theta)=-\frac{1}{2}$ if $\theta<0$.

The first relation of (A.3a) readily follows from (2.5) and the inequality $A s_{0}(\theta)>-1$ if $\theta \neq 0$ (cf. fig. 1). To obtain the other relations $(2.4)$ is differentiated twice

$$
\begin{aligned}
& s_{0}^{\prime}=B \lim _{V \rightarrow \infty} \omega^{-2} V^{\prime}(A s), \\
& s_{0}^{\prime \prime}=B \lim _{V \rightarrow \infty} \omega^{-2} V^{\prime \prime}(A s) B s_{0} .
\end{aligned}
$$

The first equality shows that $\lim _{\bar{V} \rightarrow \infty} \omega^{-2} V^{\prime}(A s)$ is a $\delta$ function. Thus $\lim _{\vec{V} \rightarrow \infty} \omega^{-2} V^{\prime \prime}(A s)$ is nonintegrable. (A.3b) follows from the second relation of (A.5) if $s_{0}$ is expressed in terms of the Heaviside function

$s_{0}(\theta)=\mp \frac{2 \pi}{2 \pi-k} H\left(\theta \mp \frac{1}{2} k\right)$ if $\quad \theta \gtrless 0$.

To calculate $\rho(\theta)$ observe that (A.2) in combination with the first relation of (A.3a) implies $\sigma^{\prime \prime}(\theta)$ $=0$, unless $\theta= \pm \frac{1}{2} k$. Correspondingly we write

$$
\begin{array}{rlrl}
\sigma(\theta) & =\sigma_{-}, & & -\pi<\theta<-\frac{1}{2} k, \\
& =\sigma_{0}, & & -\frac{1}{2} k<\theta<\frac{1}{2} k, \\
& =\sigma_{+}, \quad & \frac{1}{2} k<\theta<\pi .
\end{array}
$$

Requiring that $\rho(\theta)$ is periodic and has discontinuities only at $\theta= \pm \frac{1}{2} k$, one finds

$\sigma_{+} / \sigma_{-}=\mathrm{e}^{2 \pi \mathrm{i} \alpha}$.

To determine $\sigma(\theta)$ near $\theta=\frac{1}{2} k$, observe that the first term in the r.h.s. of (A.2) goes to zero for $\frac{1}{2} k-\varepsilon<\theta<\frac{1}{2} k+\varepsilon$, when $\bar{V} \rightarrow \infty$. The bracket part of the second term can be expressed in terms of constants $p$ and $q$ and $H(\theta)$ such that (A.2) yields

$$
\begin{aligned}
& \lim _{\hat{V} \rightarrow \infty} \sigma^{\prime \prime}\left(\hat{\theta}+\frac{1}{2} k\right)=-\lim _{\bar{V} \rightarrow \infty} \omega^{-2} m(\hat{\theta}) \\
& \quad \times\left[\frac{1}{2}(p+q)-(p-q) H(\hat{\theta})\right], \quad-\varepsilon<\hat{\theta}<\varepsilon .
\end{aligned}
$$

Integrating (A.9) twice one obtains

$p+q=0, \quad \sigma_{+}-\sigma_{0}=-\frac{1}{2}(p+q)$,

where the first equality is a consequence of the divergence of the integral in (A.3a) and the second follows with (A.3b). These relations (A.10) yield straightforwardly

$\sigma_{0}=0, \quad \sigma_{+}=\sigma_{-} \mathrm{e}^{\mathrm{i} \alpha k}$.

Together with (A.5) one finally obtains

$\alpha=\frac{N}{N-1} m, \quad m=1, \ldots, N-2$ 
and correspondingly

$\rho(\theta)=\mathrm{e}^{-\mathrm{i} \alpha \theta} \mathrm{e}^{-\mathrm{i} \alpha k / 2}, \quad-\pi<\theta<-\frac{1}{2} k$,

$\rho(\theta)=0, \quad-\frac{1}{2} k<\theta<\frac{1}{2} k$,

$\rho(\theta)=\mathrm{e}^{-\mathrm{i} \alpha \theta} \mathrm{e}^{+\mathrm{i} \alpha k / 2}, \quad \frac{1}{2} k<\theta<\pi$.

The Floquet exponents $\alpha$ (2.6a) are unique modulo one. Consequently we restrict ourselves to the values for $m$ given in (A.12).

\section{References}

[1] B.L. Holian and G.K. Straub, Phys. Rev. B 18 (1978) 1593-1608.

[2] T.J. Rolfe, S.A. Rice and J. Dancz, J. Chem. Phys. 70 (1979) 26-33.

[3] M.K. Ali and R.L. Somorjai, J. Phys. A 12 (1979) 2291-2303.

[4] Dynamical Problems in Soliton Systems, S. Takeno, ed., Proc. Seventh Kyoto Summer Institute (Springer, New York, 1985).

[5] A.R. Bishop, in [4] p. 250-257.

[6] A.R. Bishop, K. Fesser and P. Lomdahl, Physica 7D (1983) 259-279.

[7] O.H. Olsen, P. Lomdahl, A.R. Bishop and I.C. Eilbeck, J. Phys. C 18 (1985) L511-L517.
[8] P.L. Christiansen, in Chaos in Nonlinear Dynamical Systems, J. Chandra, ed. (Siam, Philadelphia, 1984) 74-85. See also [4].

[9] J. Guckenheimer and $\mathrm{Ph}$. Holmes, Nonlinear Oscillations, Dynamical Systems and Bifurcations of Vectorfields (Springer, New York, 1983).

[10] G. Iooss and D.D. Joseph, Elementary Stability and Bifurcation Theory (Springer, New York, 1980).

[11] T.P. Valkering, J. Phys. A 11 (1978) 1885-1897.

[12] T.P. Valkering, Cel. Mech. 28 (1982) 119-131.

[13] J.H. Wortel, Doctoral Report, Center for Theor. Phys., Twente Univ. Technology, Enschede, The Netherlands (1984).

[14] V.I. Karpman and V.V. Solov'ev, Physica 3D (1981) 487-502.

[15] K.A. Gorshkov and L.A. Ostrovsky, Physica 3D (1981) 428-438.

[16] A. Aceves, H. Adachihara, C. Jones, J.C. Lerman, D.W. McLaughlin, J.V. Moloney and A.C. Newell, Physica 18D (1986) 85 .

[17] N. Bekki and K. Nozaki in [4], p 268-271.

[18] K. Fesser, D.W. McLaughlin, A.R. Bishop and B.L. Holian, Phys. Rev. A 31 (1985) 2728-2731.

[19] I.I. Satija, A.R. Bishop and K. Fesser, Phys. Lett. 112A (1985) 183-187.

[20] T.P. Valkering and C. de Lange, J. Phys. A. 13 (1980) 1607-1621.

[21] D.K. Campbell, J.F. Schonfeld and C.A. Wingate, Physica 9D (1983) 1-32.

[22] W.E Ferguson, H. Flaschka and D.W. McLaughlin, J. Comp. Phys. 45 (1982) 157-209. 